\title{
In Situ Observations of Abnormal Pore Size Changes of a Zirconium Based Metal- Organic Framework Using Atomic Resolution S/TEM and EELS
}

\author{
Peter Tieu ${ }^{1}$, Chaitanya Gadre ${ }^{2}$, Wenpei $\mathrm{Gao}^{3}$, Xingxu Yan ${ }^{3}$, Muqing Li ${ }^{5}$, Zhengtao $\mathrm{Xu}^{5}$, Xiaoqing \\ $\operatorname{Pan}^{2,3,4^{*}}$ \\ 1. Department of Chemistry, University of California-Irvine, Irvine, CA 92697, USA \\ 2. Department of Physics and Astronomy, University of California-Irvine, Irvine, CA 92697, USA \\ 3. Department of Materials Science and Engineering, University of California-Irvine, Irvine, CA 92697 , \\ USA \\ 4. Irvine Materials Research Institute, University of California-Irvine, Irvine, CA 92697 \\ 5. Department of Chemistry, City University of Hong Kong, Kowloon, Hong Kong, China \\ *Corresponding author: xiaoqinp@uci.edu
}

Metal-organic frameworks (MOFs) have garnered interest due to the high surface area and uniform controlled porosity of the structure which have been used for a number of purposes including gas separation, water filtration, catalysts for the petrochemical industry, and gas storage [1]. More specific functionality, such as becoming a support for single atom catalysts and incorporating undercoordinated metal centers, often depends on additional post-synthesis modifications of the structure. Understanding the structure and the process for the post-synthesis modifications at the atomic level allows for greater control. Electron microscopy has recently been employed to understand the synthesis of MOFs as well as modifications to the structure. However, in situ works detailing the evolving structures at high spatial resolution are missing, because the organic component of MOFs can be damaged by the intense electron beam. Here, we show the combination of aberration-corrected scanning/transmission electron microscopy (S/TEM) combined with electron energy loss spectroscopy (EELS) during in situ heating offers visualization and an understanding of the structure changes of a MOF with a zirconium oxide network.

Solid MOF powder was dispersed in ethanol onto a carbon grid for electron imaging and spectroscopy. Atomic resolution STEM imaging and core loss EELS was performed on the JEOL JEM-ARM300CF at $300 \mathrm{kV}$. In situ heating was performed on a Protochips heating E-chip. Vibrational EELS was performed on the Nion UltraSTEM 200 at $60 \mathrm{kV}$ using a 50x50 phonon map using Swift's inbuilt 2D acquisition software.

In situ imaging of the $\mathrm{MOF}$ structure while heating from room temperature to $800{ }^{\circ} \mathrm{C}$ showed the lattice shrinking between 500 and $650{ }^{\circ} \mathrm{C}$. Fast Fourier transformation of the observable lattice initially showed a distance of $0.95 \mathrm{~nm}^{-1}$ between lattice points which corresponds to the distance between zirconium clusters. Upon heating to $500{ }^{\circ} \mathrm{C}$, the FFT showed an increase in the distance between lattice points. The shrinking of the lattice can be attributed to the formation of additional C-S-C and C-S-S-C bonds between the organic linkers which pulls the zirconium clusters closer together [2].

To investigate the formation of new bonds, vibrational EELS was utilized while heating the MOF structure in Nion UltraSTEM 200 at $60 \mathrm{kV}$. Raman and FTIR data provide the energy of the bonds present in the pristine MOF structure to correlate with the observed peaks in vibrational EELS. The large, broad peak around $170 \mathrm{meV}$ is attributed to the $\mathrm{C}-\mathrm{C}$ and $\mathrm{C}-\mathrm{O}$ bonds while the smaller peak around $85 \mathrm{meV}$ is the convoluted C-S and S-S bonds. Accounting for the thickness effect and the broadening of the zero loss peak, we see an increase in the ratio between the two peaks between $500-600{ }^{\circ} \mathrm{C}$, consistent with the 
presence of additional bonds at said temperatures leading to the lattice shrinking observed in the direct image and the FFT.

Using a variety of imaging and spectroscopic techniques, we are able to acquire a more comprehensive understanding of a mixed component structure as well as the changes the structure undergoes while heating. Direct, prolonged TEM observations of the lattice and the resultant FFT during heating indicate the lattice changes while vibrational EELS provides evidence of the formation of bonds that lead to the observed lattice changes. By acquiring a full picture of changes occurring in situ, we can finely control the steps during a post-synthesis modification of a material [3].

\section{References:}

[1] H. Furukawa, et al., Science 341 (2013) p. 1230444

[2] P. Gagnon, J. Boivin, D. Watson, Can. J. Chem. 37 (1959) p. 1846-1850

[3] The authors would like to acknowledge Muqing Li and Zhengtao Xu for providing the MOF sample and the corresponding Raman and FTIR data. Images and EELS data were acquired at the Irvine Materials Research Institute (IMRI).
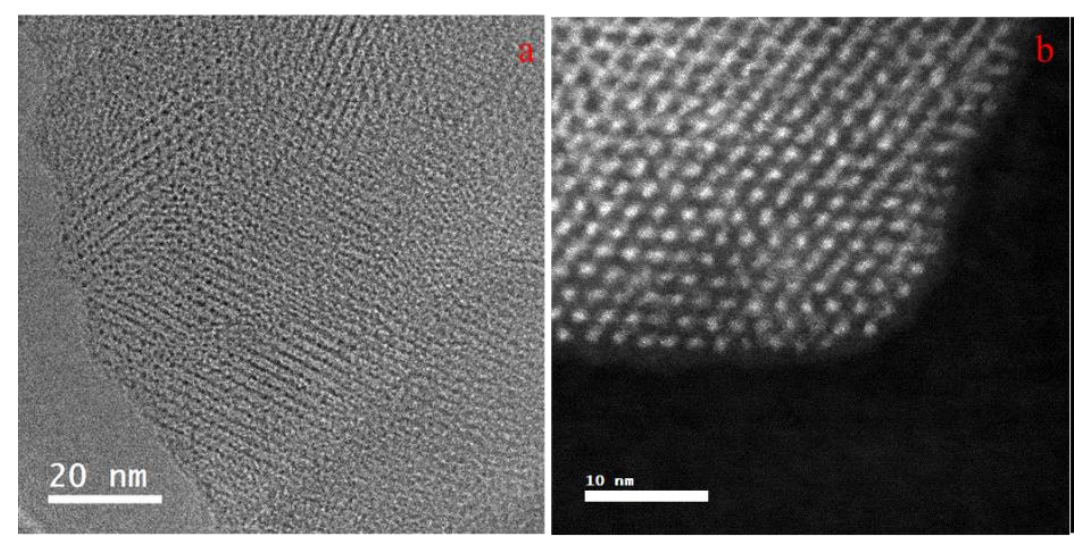

Figure 1. MOF sample imaged in (a) TEM mode at $300 \mathrm{kV}$ and (b) STEM mode at $300 \mathrm{kV}$.

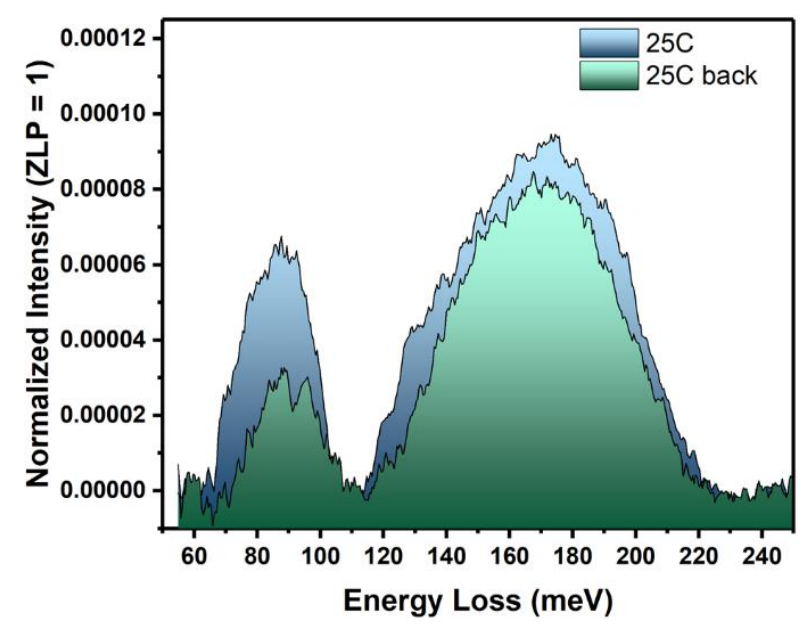

Figure 2. Vibrational EELS of the MOF before (blue) and after (green) heating showing decomposition of thiol and disulfide bonds while the aromatic rings and carbonyl moieties remain mostly unchanged. 